\section{Considerations for pharmacogenomic testing in a health system}

In their recent article, Vassy et al. described the approach of the Veterans Health Administration (VHA) Clinical Pharmacogenetics Subcommittee ("the Subcommittee") to provide recommendations for ordering individual pharmacogenetic tests to guide the prescribing of medications for which there is strong evidence for use and guidelines available from the Clinical Pharmacogenetics Implementation Consortium (CPIC; www.cpicpgx.org). ${ }^{1}$ Although each of the 30 gene/ drug pairs evaluated have CPIC recommendations of level A strength ("preponderance of evidence is high or moderate in favor of changing prescribing"), the Subcommittee determined that $4(13 \%)$ of the 30 pharmacogenetic tests should be strongly recommended before prescribing, 12 (40\%) should be recommended, and 14 (47\%) should not be routinely recommended. Reasons cited for not routinely recommending testing included if the drug was already in widespread use across the VHA and thus recommending routine testing would cause undue burden on the health-care system or if the problematic variants were relatively rare. In some cases, the reason cited was that improved patient outcomes with testing are not established, although the authors acknowledged that they did not conduct a systematic review of the literature to reach this conclusion. The premise of the Subcommittee's work rests on a reactive genotyping approach-ordering single-gene tests only when a relevant medication will be prescribed. However, the key assumption underlying the CPIC guidelines is that pharmacogenetic testing will become more widespread over time, and the pivotal question for clinical practice will not be whether to order a pharmacogenetic test, but rather what to do with the test result already in hand. With the plummeting costs of genetic sequencing and the rise of direct-to-consumer genetic testing, we are quickly approaching a future where clinicians will be routinely faced with integrating existing pharmacogenetic test results into their prescribing decisions. ${ }^{2}$

CPIC was formed in 2009 as a shared project between the Pharmacogenomics Research Network and the Pharmacogenomics Knowledgebase (PharmGKB; www.pharmgkb.org). ${ }^{3}$ CPIC brings together international clinicians and gene experts to create consensus guidelines describing how to use pharmacogenetic tests to optimize medication therapy. CPIC guidelines adhere to the National Academy of Medicine's Standards for Developing Trustworthy Clinical Practice Guidelines, and recommendations are formulated based on a systematic review of the evidence for a given gene/drug association as well as the evidence that supports alternative therapy in those with high-risk genotypes. ${ }^{3}$ The type of evidence varies and includes randomized controlled trials (RCTs) with pharmacogenetic-based prescribing versus standard care (not common for many gene/drug pairs), preclinical and clinical studies demonstrating that drug effects or concentration are linked to functional pharmacogenetic loci, case reports associating genetic variants with drug response, in vivo pharmacokinetic/pharmacodynamic studies, and in vitro metabolic and/or transport capacity studies.

RCTs are often touted as the gold standard by which clinical utility must be measured. However, with respect to pharmacogenetic associations, it is important to note that RCTs may not be necessary, practical, or ethical, and that clinical decision making based on other evidence sources is warranted and acceptable. Indeed, for many gene/drug associations, it would arguably be unethical not to act on certain pharmacogenetic test results based on available, non-RCT data. The Subcommittee deemed some of these "not routinely recommended" (e.g., CYP2C19/clopidogrel). This label could be misleading to the nonexpert, who may interpret "not routinely recommended" as "not clinically useful," when this is not the case. More than half of the CPIC actionable gene/ drug pairs are based on genetics of drug-metabolizing enzymes that have a direct impact on the pharmacokinetics of the drug, making prescribing based on genetics analogous to prescribing based on changes in liver function or renal function-a common practice based on basic pharmacologic principles, but not backed up by randomized trials.

As health-care institutions look to implement pharmacogenetic testing, pre-emptive testing for an array-based gene panel may be a more favorable and cost-effective approach compared with reactive testing. ${ }^{4-6}$ Pharmacogenetic tests have lifelong utility, and many genes may be relevant to the prescribing of several medications. Pre-emptive testing also eliminates concerns surrounding turnaround time for results, as test results are already available in the electronic health record at the point of prescribing. Studies from centers with established pre-emptive pharmacogenetic testing services have shown that the great majority of patients harbor at least one high-risk variant that warrants a change from usual prescribing to avoid an undesirable drug response. ${ }^{7-9}$ A recent study of pharmacogenetic testing in patients with polypharmacy over the age of 50 , which is likely generalizable to the VHA population, showed that using the test results to optimize therapy reduced the rate of rehospitalizations and emergency department visits compared with controls who received standard care. ${ }^{10}$ For some gene/drug pairs that the Subcommittee labeled as "not routinely recommended," they include an annotation noting that testing may be considered 
for patients who have already experienced toxicity (e.g., fluoropyrimidines) or therapeutic failure (e.g., clopidogrel). However, this approach undermines the promise of pharmacogenetic testing, which is to avoid untoward drug responses in the first place. Using pharmacogenetic test results up front to guide therapy will yield the most value for the patient.

In summary, CPIC guidelines provide evidence-based prescribing recommendations for gene/drug pairs, many of which are supported by a high level of evidence. These guidelines are developed with the underlying assumption that the clinician already has access to the patient's pharmacogenetic test results. Assessments of clinical utility that assume a model of single-gene, reactive testing are not consistent with the coming era of broad pre-emptive genomic testing.

\section{ACKNOWLEDGEMENTS}

The authors are funded by the National Institutes of Health $(\mathrm{NIH})$ for CPIC (R24GM115264 and U24HG010135) (R.S.G., K.E.C., T. E.K., M.V.R.) and PharmGKB (R24GM61374) (T.E.K.).

\section{DISCLOSURE}

The authors declare no conflicts of interest.

Publisher's note: Springer Nature remains neutral with regard to jurisdictional claims in published maps and institutional affiliations.

\section{Roseann S. Gammal, PharmD (iD ${ }^{1,2}$, Kelly E. Caudle, PharmD, PhD ${ }^{2}$, Teri E. Klein, $P h D^{3}$ and Mary V. Relling, PharmD ${ }^{2}$}

${ }^{1}$ Department of Pharmacy Practice, MCPHS University School of Pharmacy, Boston, MA, USA; ${ }^{2}$ Department of Pharmaceutical Sciences, St. Jude Children's Research Hospital, Memphis, TN, USA; ${ }^{3}$ Department of Biomedical Data Science, Stanford
University, Stanford, CA, USA. Correspondence: Roseann S. Gammal (roseann. gammal@mcphs.edu)

\section{REFERENCES}

1. Vassy JL, Stone A, Callaghan JT, et al. Pharmacogenetic testing in the Veterans Health Administration (VHA): policy recommendations from the VHA Clinical Pharmacogenetics Subcommittee. Genet Med. 2018 Jun 1; doi:10.1038/s41436-018-0057-x [Epub ahead of print].

2. National Human Genome Research Institute (NHGRI). DNA sequencing costs: data. https://www.genome.gov/27541954/dna-sequencing-costsdata/. Accessed 1 October 2018.

3. Caudle KE, Klein TE, Hoffman JM, et al. Incorporation of pharmacogenomics into routine clinical practice: the Clinical Pharmacogenetics Implementation Consortium (CPIC) guideline development process. Curr Drug Metab. 2014;15:209-217.

4. Weitzel KW, Cavallari LH, Lesko LJ. Preemptive panel-based pharmacogenetic testing: the time is now. Pharm Res. 2017; 34:1551-1555.

5. Roden DM, Van Driest SL, Mosley JD, et al. Benefit of preemptive pharmacogenetic information on clinical outcome. Clin Pharmacol Ther. 2018;103:787-794

6. Dunnenberger HM, Crews KR, Hoffman JM, et al. Preemptive clinical pharmacogenetics implementation: current programs in five US medical centers. Annu Rev Pharmacol Toxicol. 2015;55:89-106.

7. Van Driest SL, Shi Y, Bowton EA, et al. Clinically actionable genotypes among 10,000 patients with preemptive pharmacogenomic testing. Clin Pharmacol Ther. 2014;95:423-431.

8. Hoffman JM, Haidar CE, Wilkinson MR, et al. PG4KDS: a model for the clinical implementation of pre-emptive pharmacogenetics. Am J Med Genet C Semin Med Genet. 2014;166C:45-55.

9. O'Donnell PH, Danahey K, Ratain MJ. The outlier in all of us: why implementing pharmacogenomics could matter for everyone. Clin Pharmacol Ther. 2016;99:401-404.

10. Elliott LS, Henderson JC, Neradilek MB, Moyer NA, Ashcraft KC, Thirumaran RK. Clinical impact of pharmacogenetic profiling with a clinical decision support tool in polypharmacy home health patients: a prospective pilot randomized controlled trial. PLOS ONE. 2017;12: e0170905.

Advance online publication 9 January 2019. doi:10.1038/s41436-018-0421-x 